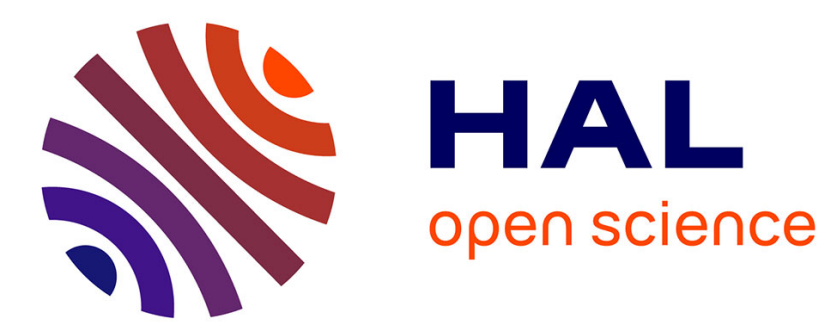

\title{
Thermal-Wave Diode
}

Jose Ordonez-Miranda, Yangyu Guo, Juan J Alvarado-Gil, Sebastian Volz, Masahiro Nomura

\section{To cite this version:}

Jose Ordonez-Miranda, Yangyu Guo, Juan J Alvarado-Gil, Sebastian Volz, Masahiro Nomura. Thermal-Wave Diode. Physical Review Applied, 2021, 16 (4), pp.L041002. 10.1103/physrevapplied.16.1041002 . hal-03420124

\section{HAL Id: hal-03420124 \\ https://hal.science/hal-03420124}

Submitted on 9 Nov 2021

HAL is a multi-disciplinary open access archive for the deposit and dissemination of scientific research documents, whether they are published or not. The documents may come from teaching and research institutions in France or abroad, or from public or private research centers.
L'archive ouverte pluridisciplinaire HAL, est destinée au dépôt et à la diffusion de documents scientifiques de niveau recherche, publiés ou non, émanant des établissements d'enseignement et de recherche français ou étrangers, des laboratoires publics ou privés. 


\title{
Thermal-Wave Diode
}

\author{
Jose Ordonez-Miranda $\odot,{ }^{1,2, *}$ Yangyu Guo $\odot,{ }^{2}$ Juan J. Alvarado-Gilø ${ }^{3}$ Sebastian Volz $\odot,{ }^{1,2}$ \\ and Masahiro Nomura $\oplus^{1,2}$ \\ ${ }^{1}$ LIMMS, CNRS-IIS UMI 2820, The University of Tokyo, Tokyo 153-8505, Japan \\ ${ }^{2}$ Institute of Industrial Science, The University of Tokyo, Tokyo 153-8505, Japan \\ ${ }^{3}$ Applied Physics Department, CINVESTAV-IPN, Mérida, Yucatán 97310, Mexico
}

(Received 22 February 2021; revised 24 September 2021; accepted 8 October 2021; published 28 October 2021)

\begin{abstract}
Based on the spatiotemporal modulation of thermal conductivity and volumetric heat capacity, we propose a thermal-wave diode characterized by the rectification of the heat currents carried by thermal waves. By transforming Fourier's law for the heat flux and the diffusion equation for the temperature into equations with constant coefficients, it is shown that: (i) the rectification effect is generated by the simultaneous wavelike modulation of both thermal properties, such that it disappears in the absence of either of them, and (ii) the rectification factor can be optimized and tuned by means of the speed and phase difference of the variations of the heat capacity and thermal conductivity. High rectification factors, greater than $86 \%$, are obtained for lower frequencies driving the propagation of thermal waves. The proposed thermal-wave diode is thus analogous to its electronic counterpart operating with modulated electrical currents and can open a vista for developing different types of thermal-wave logic components.
\end{abstract}

DOI: 10.1103/PhysRevApplied.16.L041002

Nonlinear heat transport based on the metal-insulator transition of materials [1] and the coupling of dissimilar lattices [2-5] has recently attracted great interest for the control of conductive and radiative heat currents [6-10]. This phenomenon is observed in materials with thermal and optical properties depending on temperature, which has been exploited to conceive and develop thermal diodes [6,11-17], thermal transistors [18-21], thermal memories $[22,23]$, and thermal memristors [24,25] that make possible the processing of information via thermal electrons, phonons, and photons. The operation of these thermal devices is, however, usually limited temperatures in which the material properties exhibit significant changes with temperature. Heat control with nonlinear materials is thus typically restricted to narrow temperature ranges.

Materials with properties periodically modulated in space and time are able to overcome the major drawbacks of nonlinear ones, given that they are able to operate at required temperature, frequency, and scale [26,27]. This spatiotemporal modulation of material properties has been theoretically and experimentally studied in elastic $[8,28,29]$, acoustic [30-33], photonic [34,35], and thermal [36-39] systems. As a result of the wavelike variation of their properties, these materials exhibit a nonreciprocal response for the propagation of waves, which provides an effective method to allow the flow of energy in a given direction and impede it in the opposite one. By considering

\footnotetext{
*jose.ordonez@cnrs.fr
}

the spatiotemporal modulation of thermal conductivity and heat capacity in the form of a traveling wave, Torrent et al. [39] showed that a material behaves like an effective medium with constant thermal properties and an internal convectionlike term in the diffusion equation describing its temperature profile. This convective term induces the nonreciprocal response of the material for heat flux, which takes different values depending on its direction parallel or antiparallel to the traveling waves of the thermal properties. Those authors thus demonstrated that materials with thermal conductivity and heat capacity periodically modulated in space and time can be used to develop thermal diodes operating with steady-state heat fluxes [39]. As this modulation of the thermal properties has a dynamic origin, its impact on the transient heat conduction regime is expected to be strong, but has not been studied yet. In particular, given that this dynamic regime is driven by the heat capacity, its spatiotemporal modulation may induce a nonreciprocal behavior on the heat flux carried by the thermal waves predicted by Fourier's law [40]. These diffusion waves are periodic temperature fluctuations widely applied in thermal detection via photothermal and photoacoustic techniques [40-42] that could be implemented to generate and detect the rectification of their heat currents.

The purpose of this Letter is to demonstrate the proof of principle of a thermal-wave diode based on the spatiotemporal modulation of thermal conductivity and volumetric heat capacity. This is done by determining and optimizing the rectification of the heat fluxes carried by the nonreciprocal thermal waves propagating in the forward and 
(a)

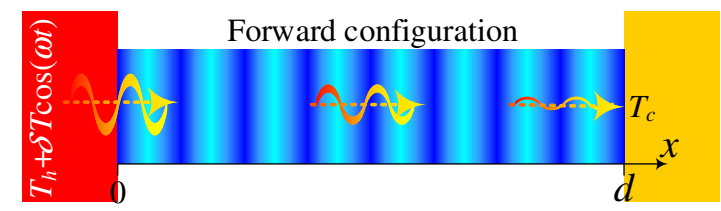

(b)

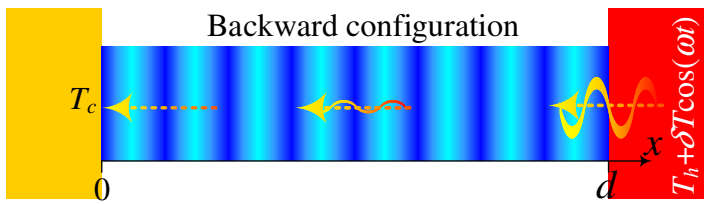

FIG. 1. Scheme of a thermal-wave diode operating in the (a) forward and (b) backward configurations. Arrows along with their amplitudes indicate the propagation direction and intensity of the thermal waves generated by the excitation $\delta T \cos (\omega t)$.

backward bias. In contrast to steady-state thermal diodes, which typically operate with large temperature gradients $[16,17]$, this thermal-wave diode functions with relatively small heat currents modulated in frequency.

Let us consider two thermal baths exchanging heat by conduction through a nonreciprocal material, due to their temperature difference $T_{h}-T_{c}+\delta T \cos (\omega t)$ periodically modulated in time $t$ with frequency $\omega$, as shown in Figs. 1(a) and 1(b). In the forward configuration [Fig. 1(a)], the heat flux $q_{F}$ is driven from left to right $\left(T_{h}>T_{c}\right)$, while in the backward one [Fig. 1(b)], the heat flux $q_{B}$ appears in the opposite direction. The magnitudes of $q_{F}$ and $q_{B}$ are expected to be different due to the periodic modulation in space $x$ and time $t$ of the material conductivity $k=k(x-v t)$ and volumetric heat capacity $C=$ $C(x-v t)$, which are known to induce nonreciprocal heat transport [39]. These thermal properties thus behave like waves traveling at speed $v$ and are periodic functions of $x-v t$ with period $a$. The working principle of the proposed diode is hence based on the propagation of thermal waves driven by the spatiotemporal modulation of thermal properties, which is different from that of steady-state diodes based on the temperature dependence of thermal conductivity [11] and nonlinear lattices [6]. Given that $q_{F}$ and $q_{B}$ will generally depend on position $x$, the ability of our thermal-wave diode to rectify heat currents can be defined by the following rectification factor $R[6,11]$ :

$$
R=\frac{|| q_{F}(x=d)|-| q_{B}(x=0)||}{\max \left[\left|q_{F}(x=d)\right| ;\left|q_{B}(x=0)\right|\right]} .
$$

The optimal rectification $(R \rightarrow 1)$ is thus achieved when the reversal of the thermal gradient induces a sizable variation of the heat flux magnitude. Considering that the diode length $d$ and period $a$ are much longer than the mean free path (approximately $1-10^{3} \mathrm{~nm}$ [40]) of the material heat carriers and that the heat flux $q$ is determined at a time much longer than their relaxation time (approximately $1-10^{3}$ ps [40]), $q$ in each configuration is given by Fourier's law $q=-k(x-v t) \partial T / \partial x$, where the temperature $T$ is described by the diffusion equation $[39,43]$

$$
\frac{\partial}{\partial x}\left(k(x-v t) \frac{\partial T}{\partial x}\right)=C(x-v t) \frac{\partial T}{\partial t} .
$$

The wavelike modulation of $k$ and $C$ can be implemented by means of materials that can be switched between low and high thermal conductivity and heat capacity states, as is the case for the azobenzene polymer $[44,45]$. Under UV $(375 \mathrm{~nm})$ and green $(530 \mathrm{~nm})$ light illuminations, this light-responsive material undergoes a fully reversible transition of its thermal properties that occurs in a few seconds at room temperature [44]. The periodic illumination with UV and green light of a layered system made up of azobenzene polymer layers separated by thermally insulating plates can efficiently be used to generate the modulation of $k$ and $C$ (see Supplemental Material [46] for details). $k$ and $C$ can then be written as $[28,39]$

$$
\begin{aligned}
k(x-v t) & =k_{0}\left\{1+\Delta_{k} \cos [\sigma(x-v t)]\right\}, \\
C(x-v t) & =C_{0}\left\{1+\Delta_{c} \cos [\sigma(x-v t+\varphi)]\right\},
\end{aligned}
$$

where $\sigma=2 \pi / a, k_{0}$ and $C_{0}$ are the respective thermal conductivity and volumetric heat capacity in the absence of modulation, $\Delta_{k}$ and $\Delta_{c}$ are the modulation amplitudes of $k$ and $C$, and $\varphi$ is a phase shift that we introduce to tune the thermal response and rectification factor of the proposed diode, as shown below. Given that Eq. (2) is a partial differential equation with oscillatory coefficients, it can be replaced by a homogenized differential equation with constant coefficients, as established by the homogenization theory of differential equations [43]. When the spatial variations of $T$ are larger than the period $a$ of $k$ and $C$, the average temperature $\bar{T}$ and heat flux $\bar{q}$ are given by [46]

$$
\begin{aligned}
\frac{\partial^{2} \bar{T}}{\partial x^{2}} & =\frac{1}{\alpha} \frac{\partial \bar{T}}{\partial t}+\frac{1}{L} \frac{\partial \bar{T}}{\partial x}+\frac{1}{V} \frac{\partial^{2} \bar{T}}{\partial x \partial t}, \\
\bar{q} & =-k^{*}\left(\frac{\partial \bar{T}}{\partial x}+\frac{1}{2 V} \frac{\partial \bar{T}}{\partial t}\right),
\end{aligned}
$$

where the effective thermal conductivity $k^{*}$ and thermal diffusivity $\alpha$ along with the characteristic length $L$ and speed $V$ are defined as follows:

$$
\begin{aligned}
k^{*} & =k_{0}\left(1-\frac{0.5 \Delta_{k}^{2}}{1+\Gamma^{2}}\right), \\
\alpha^{-1} & =\frac{1}{\alpha_{0}} \frac{1+\Gamma^{2}-0.5\left(\Delta_{c} \Gamma\right)^{2}}{1+\Gamma^{2}-0.5 \Delta_{k}^{2}},
\end{aligned}
$$




$$
\begin{aligned}
L^{-1} & =\frac{\sigma \Delta_{k} \Delta_{c}}{2} \frac{\Gamma[\cos (\varphi)+\Gamma \sin (\varphi)]}{1+\Gamma^{2}-0.5 \Delta_{k}^{2}} \\
V^{-1} & =\frac{\Delta_{k} \Delta_{c}}{\sigma \alpha_{0}} \frac{\sin (\varphi)+\Gamma \cos (\varphi)}{1+\Gamma^{2}-0.5 \Delta_{k}^{2}}
\end{aligned}
$$

with $\Gamma=v C_{0} / \sigma k_{0}=v / \sigma \alpha_{0}$ and $\alpha_{0}=k_{0} / C_{0}$ being the normalized speed of modulation and intrinsic thermal diffusivity of the considered material, respectively. Equation (4a) establishes that the averaged temperature is driven by a conduction-convection-like equation with an additional term $\left(V^{-1}\right)$ analogous to the Willis coefficient found in elastodynamics of inhomogeneous media [47,48]. According to Eqs. (4a) and (4b), this term is relevant to the transient (thermal wave) heat conduction regime considered in this work and appears due to the spatiotemporal modulation of both $k$ and $C$, as indicated in Eq. (5d). Note that $\bar{q}$ involves not only a spatial derivative of temperature, as is the case for Fourier's law, but also a time derivative, which leads to interesting thermal phenomena. In the absence of modulation $\left(\Delta_{k}=\Delta_{c}=0=\Gamma\right)$, $k=k_{0}, \alpha=\alpha_{0}, L \rightarrow \infty$, and $V \rightarrow \infty$ and Eqs. (4a) and (4b) reduce to their standard counterparts, as expected. In the presence of modulation, $k^{*}$ and $\alpha$ are both independent of $\varphi$ and even functions of $\Gamma$, which means that reversing the direction of the modulation has no effect on their values. By contrast, the dependence of $L$ and $V$ on $\Gamma$ is driven by $\varphi$. For instance, when $\varphi=0$, both $L$ and $V$ are odd functions of $\Gamma$, while they become even functions of $\Gamma$ for $\varphi=\pi / 2$. The nonreciprocal thermal response of the material can thus be activated, nullified, or tuned by means of the characteristic length and speed with $\varphi=0, \varphi=\pi / 2$, or $0<\varphi<\pi / 2$, respectively. Note that the reciprocity is recovered not only in the absence of the traveling modulation $(\Gamma=0)$, but also for very high speeds $(\Gamma \rightarrow \pm \infty)$. In this latter case, the oscillations of $k=k(x-v t)$ and $C=C(x-v t)$ are so fast that their spatial variations tend to disappear and hence their spatiotemporal modulation reduces to a temporal one. This is the reason why the modulation effect appears (disappears) for $\alpha=\alpha_{0} /\left(1-0.5 \Delta_{c}^{2}\right)$ (conductivity $\left.k^{*}=k_{0}\right)$ that drives the time (spatial) variations of temperature, as established by Eq. (4a). It is thus clear that there exists an optimal speed $\Gamma$ that maximizes the material nonreciprocity, as shown below. Note that the coefficient $\left(L^{-1}\right)$ of the convective term in Eq. (4a) is generated by the coupling of the modulation of $k$ and $C$, which indicates that this term of dynamic origin (via $C$ ) has a direct impact on the average temperature $\bar{T}$, even under steady-state conditions, as predicted by Eq. (4a).

Taking into account that the operation of the thermalwave diode depicted in Fig. 1 is driven by the temperature difference $T_{h}-T_{c}+\delta T \operatorname{Re}\left(e^{i \omega t}\right)$ modulated in time and that Eq. (4a) is a linear differential equation, the general solutions for $\bar{T}$ and $\bar{q}$ have the following forms:

$$
\begin{aligned}
\bar{T}(x, t) & =\psi(x)+\operatorname{Re}\left[\theta(x) e^{i \omega t}\right], \\
\bar{q}(x, t) & =Q_{\mathrm{SS}}(x)+\operatorname{Re}\left[Q(x) e^{i \omega t}\right],
\end{aligned}
$$

where the steady-state $\left(\psi, Q_{\mathrm{Ss}}\right)$ and modulated $(\theta, Q)$ components are associated with $T_{h}-T_{c}$ and $\delta T$, respectively. In practice, the temperature oscillations of the hotter thermal bath can be set with a Peltier cell, while the other bath is kept at constant temperature with a second Peltier cell. These cells typically operate with a resolution better than $0.1 \mathrm{~K}$ and modulation frequencies from 0.1 to $100 \mathrm{~Hz}$ [49]. According to Fig. $1,[\psi(0), \psi(d)]=\left(T_{h}, T_{c}\right)$ and $[\theta(0), \theta(d)]=(\delta T, 0)$ for the forward configuration, while for the backward one $[\psi(0), \psi(d)]=\left(T_{c}, T_{h}\right)$ and $[\theta(0), \theta(d)]=(0, \delta T)$. After inserting Eq. (6a) into Eq. (4a), one obtains ordinary differential equations for $\psi$ and $\theta$, whose solutions for the two diode configurations are

$$
\begin{aligned}
& \psi_{F}(x)=\frac{T_{h} e^{d / L}-T_{c}-\left(T_{h}-T_{c}\right) e^{x / L}}{e^{d / L}-1}, \\
& \psi_{B}(x)=\frac{T_{c} e^{d / L}-T_{h}+\left(T_{h}-T_{c}\right) e^{x / L}}{e^{d / L}-1}, \\
& \theta_{F}(x)=\delta T \frac{e^{-(d-x) \sigma_{-}}-e^{-(d-x) \sigma_{+}}}{e^{-d \sigma_{-}}-e^{-d \sigma_{+}}}, \\
& \theta_{B}(x)=\delta T \frac{e^{x \sigma_{+}}-e^{x \sigma_{-}}}{e^{d \sigma_{+}}-e^{d \sigma_{-}}}
\end{aligned}
$$

where the wave numbers $\sigma_{ \pm}=1 / 2 L \pm \chi_{+}+i\left(1 / r \pm \chi_{-}\right)$ are determined by the three lengths $L, r=2 V / \omega$, and $\mu=\sqrt{2 \alpha / \omega}$ involved in $\chi_{ \pm}=\sqrt{\sqrt{y^{2}+z^{2}} \pm y}$, with $y=0.5\left[(2 L)^{-2}-r^{-2}\right]$ and $z=\mu^{-2}+1 / 2 r L$. Furthermore, according to Eqs. (4b), (6b), and (7), the steady-state $\left(Q_{\mathrm{SS}}=-k^{*} d \psi / d x\right)$ and modulated $[Q=$ $\left.-k^{*}(d \theta / d x+i \theta / r)\right]$ components of the heat flux read

$$
\begin{aligned}
Q_{\mathrm{SS}, F} & =-Q_{\mathrm{SS}, B}=k^{*} \frac{\left(T_{h}-T_{c}\right)}{L} \frac{e^{x / L}}{e^{d / L}-1} \\
\frac{Q_{F}}{k^{*} \delta T} & =\frac{\left(\sigma_{+}+i / r\right) e^{-(d-x) \sigma_{+}}-\left(\sigma_{-}+i / r\right) e^{-(d-x) \sigma_{-}}}{e^{-d \sigma_{-}}-e^{-d \sigma_{+}}} \\
\frac{Q_{B}}{k^{*} \delta T} & =\frac{\left(\sigma_{-}+i / r\right) e^{x \sigma_{-}}-\left(\sigma_{+}+i / r\right) e^{x \sigma_{+}}}{e^{d \sigma_{+}}-e^{d \sigma_{-}}}
\end{aligned}
$$

The exponential dependence on position of the temperatures in Eqs. (7a) and (7b) reduces to the usual linear one for $d \ll L$. The steady-state heat fluxes in Eq. (8a), on the other hand, depend on position due to the spatiotemporal modulation of $k$, such that they reduce to their usual constant $\left[Q_{\mathrm{SS}, F}=-Q_{\mathrm{SS}, B}=k^{*}\left(T_{h}-T_{c}\right) / d\right]$ in the absence of modulation $(L \rightarrow \infty)$, as expected. Note that $Q_{\mathrm{SS}, F}$ and 
$Q_{\mathrm{SS}, B}$ are different at an equivalent position with respect to the thermal excitation ( $x$ for $Q_{\mathrm{SS}, F}$ and $d-x$ for $Q_{\mathrm{SS}, B}$ ), and hence, according to Eqs. (1) and (8a), the rectification factor of the steady-state heat fluxes is given by

$$
R=1-e^{-d / L} .
$$

Equation (9) is consistent with the result obtained by Torrent et al. [39], for a pure steady-state problem, and shows that for a given material length $d$, the optimal rectification factor is given by the maximum of $L^{-1}$ [46]: $\left(L^{-1}\right)_{\max }=$ $\left(\sigma \Delta_{k} \Delta_{c} / 4\right)\left[\sin (\varphi)+\sqrt{\sin ^{2}(\varphi)+\lambda^{-1} \cos ^{2}(\varphi)}\right]$, with $\lambda$ $=1-0.5 \Delta_{k}^{2}$. This latter expression thus confirms that the rectification of the steady-state heat fluxes can be tuned with the phase shift $\varphi$ and increases with the spatiotemporal variation factor $\Delta_{k} \Delta_{c}$ of $k$ and $C$.

The modulated temperature and heat flux profiles are thermal waves whose spatial attenuation and propagation are, respectively, determined by the real and imaginary parts of the wave numbers $\sigma_{ \pm}$, as established by Eqs. (7c), $(7 d),(8 b)$, and (8c). In the limit of no spatiotemporal variations $\left(\Delta_{k}=\Delta_{c}=0=\Gamma\right)$, these wave numbers reduce to $\sigma_{ \pm}= \pm(1+i) / \mu_{0}$, which are their standard expressions with $\mu_{0}=\sqrt{2 \alpha_{0} / \omega}$ being the classical diffusion length of the thermal waves propagating along the $\mp x$ directions [42]. In the presence of the spatiotemporal modulation, Eqs. (6)-(8) establish that the thermal waves in the forward and backward configurations are driven by $\exp \left[x \sigma_{ \pm}\right]=$ $\exp \left\{x\left(1 / 2 L \pm \chi_{+}\right)+i\left[\omega t+x\left(1 / r \pm \chi_{-}\right)\right]\right\}$, which indicates that $\sigma_{+}\left(\sigma_{-}\right)$is related to a wave propagating in the $-x(+x)$ direction, given that $1 / r<\chi_{-}$. For both configurations, the propagation length of the thermal waves depends on the direction and is defined by $\Lambda_{ \pm}=$ $\left(\chi_{+} \pm 1 / 2 L\right)^{-1}$. As $\chi_{+}$and $L$ are, in general, not even functions of $\Gamma$, this latter relation establishes that the propagation of the thermal waves can be tuned by changing the modulation direction, that is to say, by replacing $\Gamma$ by $-\Gamma$. The propagation length $\Lambda_{-}\left(\Lambda_{+}\right)$is typically greater (smaller) than the diffusion length $\mu_{0}$, such that its maximum (minimum) value shows up at a speed $\Gamma$ that increases with the phase shift $\varphi$, as shown in Fig. 2(a). Calculations for this and other figures are done for the typical amplitudes [28,39] $\Delta_{k}=2 \Delta_{c}=2 / 3$ and $d=5 a$. The fact that $\Lambda_{-}>d$ and $\Lambda_{+}<d$ indicates that the thermal-wave energy in both configurations is mainly determined by the thermal waves propagating in the direction parallel to the wavelike modulation of the material thermal properties. At very low speeds $(\Gamma \rightarrow 0)$, the modulation effect disappears and both propagation lengths reduce to the standard diffusion length of thermal waves $\left(\Lambda_{ \pm}=\mu_{0}\right)$, as shown by the dashed line in Fig. 2(a). For $f=f_{c}, \mu_{0}=d \sqrt{1-0.5 \Delta_{k}^{2}}<$ $d$ and hence $\Lambda_{ \pm} / d$ converges to values smaller than unity for $\Gamma \rightarrow 0$. More importantly, the fact that $\Lambda_{+} \neq \Lambda_{-}$, for $\Gamma>0$, induces deviations from the mirrorlike behavior of (a)

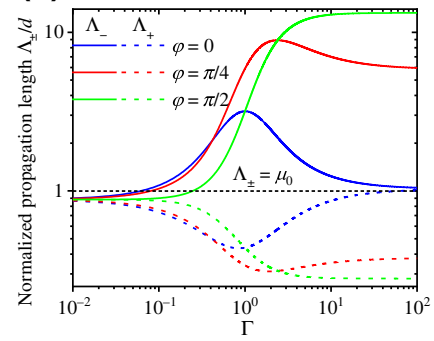

(b)

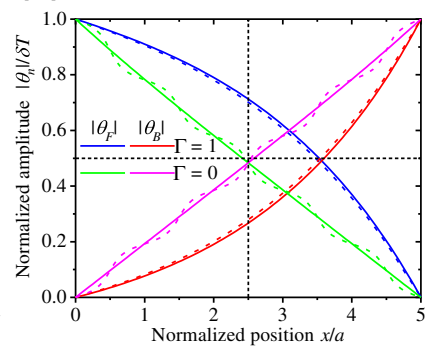

FIG. 2. (a) Propagation length of a thermal wave propagating in the $+x\left(\Lambda_{+}\right)$and $-x\left(\Lambda_{-}\right)$directions as a function of the normalized speed $\Gamma$, for three phase shifts. (b) Amplitude profiles of the temperature field for the forward and backward configurations. Calculations are done for $f=f_{c}=\alpha_{0} / \pi d^{2}$ and the dashed lines in (b) represent the numerical solution of Eq. (2) obtained through the finite difference method (FDM) [46].

the temperature profiles around $x=5 a / 2=d / 2$ and $\theta_{n}=$ $\delta T / 2$, as shown in Fig. 2(b) for the forward $(n=F)$ and backward $(n=B)$ configurations. This asymmetric behavior of $\theta_{n}$ is expected to give rise to the rectification effect of the thermal-wave heat fluxes.

Based on the amplitudes of the thermal-wave heat fluxes, Eq. (1) takes the form $R=1-\left|Q_{B}(0)\right| /\left|Q_{F}(d)\right|$, which exactly reduces to Eq. (9), as established by Eqs. (8b) and (8c). This indicates that the rectification of the heat fluxes at the arrival positions $\left(x=d\right.$ for $Q_{F}$ and $x=0$ for $Q_{B}$ ) is independent of the modulation frequency and its maximum occurs at the highest value of $L^{-1}$. By contrast, in terms of the heat fluxes at the excitation (departure) positions, the rectification factor in Eq. (1) reads $R_{d}=$ $1-\left|Q_{B}(d)\right| /\left|Q_{F}(0)\right|$, which yields

$$
\begin{aligned}
& R_{d} \\
& =1-\left|\frac{(1 / 2 L+2 i / r) \tanh \left[\left(\chi_{+}+i \chi_{-}\right) d\right]-\left(\chi_{+}+i \chi_{-}\right)}{(1 / 2 L+2 i / r) \tanh \left[\left(\chi_{+}+i \chi_{-}\right) d\right]+\left(\chi_{+}+i \chi_{-}\right)}\right|
\end{aligned}
$$

Taking into account the proportionality of $L^{-1}$ and $r^{-1}$ to the product $\Delta_{k} \Delta_{c}$, Eq. (10) establishes that the rectification $(R>0)$ of the thermal-wave heat fluxes is generated by the simultaneous spatiotemporal modulation of both $k$ and $C$, such that it disappears in the absence of either of them $\left(R_{d}=0\right.$ for $\left.\Delta_{k} \Delta_{c}=0\right)$. In the low-frequency limit ( $\left.f=\omega / 2 \pi \ll f_{c}=\alpha_{0} / \pi d^{2}\right)$, Eq. (10) reduces to Eq. (9), as expected for the steady-state regime.

The predictions of Eqs. (8b) and (8c) for the heat flux amplitudes at the arrival and departure positions are in good agreement with their corresponding numerical counterparts, as shown in Figs. 3(a) and 3(b), respectively. Note that, at the arrival position $x=d(x=0)$, the amplitude $A_{F}\left(A_{B}\right)$ follows the behavior of $\Lambda_{-}\left(\Lambda_{+}\right)$, as shown in Fig. 2(a). The opposite applies for the amplitudes 
(a)

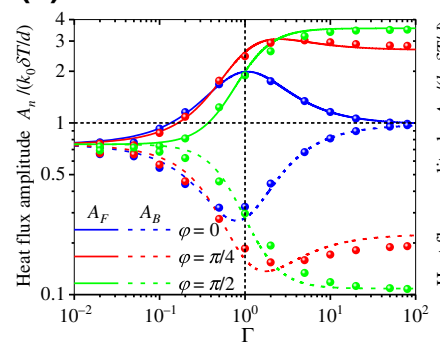

(b)

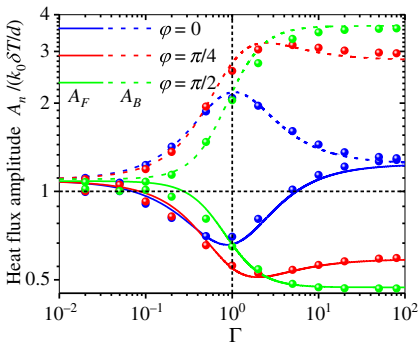

FIG. 3. Speed $\Gamma$ dependence of the heat flux amplitudes at the (a) arrival and (b) departure positions for the forward and backward configurations. Calculations are done for $f=f_{c}$ and three representative phase shifts. Points represent the numerical solution of Eq. (2) obtained using the FDM [46].

at the departure positions, as shown in Fig. 3(b). For both positions, there exists an optimal normalized speed $\Gamma_{\text {opt }} \gtrsim 1$ that increases with the phase shift $\varphi$, for which the amplitude difference $\left|A_{F}-A_{B}\right|$ takes its highest value. This indicates that the rectification factor is maximized at $\Gamma_{\text {opt }}=\lambda \tan (\varphi)+\lambda \sqrt{\tan ^{2}(\varphi)+\lambda^{-1}}$, as predicted by Eq. (9), and is shown in Fig. 4(a) for the rectification of the heat fluxes at the arrival positions. For the departure positions, on the other hand, $R_{d}$ exhibits a dependence on $\Gamma$ similar to that of $R$; however, its values decrease as the thermal-wave frequency increases, as displayed in Fig. 4(b). When $\varphi$ is changed from 0 to $\pi / 2, R_{d}$ takes lower values for $\Gamma=0.1$, equal values for $\Gamma=1$, and higher values for $\Gamma=10$. The fact that the same propagation lengths [Fig. 2(a)], heat fluxes (Fig. 3), and rectification factors [Fig. 4(a)] can be obtained by different sets of values of $\Gamma$ and $\varphi$ is because the dependences of $L$ and $V$ on $\Gamma$ are driven by the particular values of $\varphi$. This phase shift thus provides a degree of freedom to optimize the diode rectification factor.

In summary, the proof of principle of a thermal-wave diode is demonstrated by exploiting the spatiotemporal modulation of the thermal conductivity and volumetric heat capacity in a wavelike fashion. This is done by deriving explicit expressions for the average temperature and heat flux carried by the thermal waves propagating in the forward and backward configurations of the diode. It is shown that the rectification effect is generated by the simultaneous spatiotemporal variations of both the heat capacity and thermal conductivity, such that their modulation speed and phase difference drive the diode rectification factor. The highest rectification factor, greater than $86 \%$, is reached at low modulation frequencies and an optimal speed that increases with the phase shift. Considering the unique features of the proposed thermal-wave diode, the developed theory and our findings can open research avenues in thermal computing, unconventional thermal phenomena, and material science.

Acknowledgments. This work is supported by the Postdoctoral Fellowship of Japan Society for the Promotion of (a)

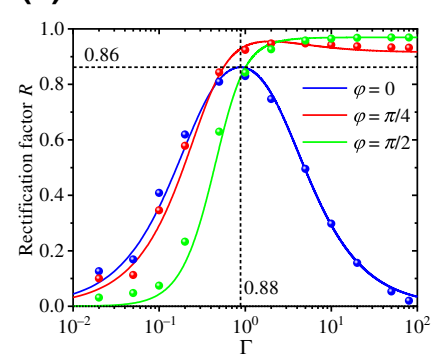

(b)

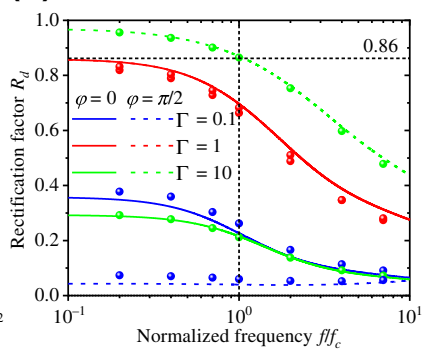

FIG. 4. Rectification factors for the heat fluxes carried by thermal waves at the (a) arrival and (b) departure positions, as functions of the normalized speed and frequency, respectively. Points represent the FDM numerical solution of Eq. (2) [46].

Science, Grant No. P19353, and CREST Japan Science and Technology Agency, Grants No. JPMJCR19Q3 and No. JPMJCR19I1.

[1] J. Zhu, K. Hippalgaonkar, S. Shen, K. Wang, Y. Abate, S. Lee, J. Wu, X. Yin, A. Majumdar, and X. Zhang, Temperature-gated thermal rectifier for active heat flow control, Nano Lett. 14, 4867 (2014).

[2] C. W. Chang, D. Okawa, A. Majumdar, and A. Zettl, Solidstate thermal rectifier, Science 314, 1121 (2006).

[3] H. Wang, S. Hu, K. Takahashi, X. Zhang, H. Takamatsu, and J. Chen, Experimental study of thermal rectification in suspended monolayer graphene, Nat. Comm. 8, 15843 (2017).

[4] R. Shrestha, Y. Luan, X. Luo, S. Shin, T. Zhang, P. Smith, W. Gong, M. Bockstaller, T. Luo, R. Chen, K. Hippalgaonkar, and S. Shen, Dual-mode solid-state thermal rectification, Nat. Comm. 11, 4346 (2020).

[5] Y. Wang, A. Vallabhaneni, J. Hu, B. Qiu, Y. P. Chen, and X. Ruan, Phonon lateral confinement enables thermal rectification in asymmetric single-material nanostructures, Nano Lett. 14, 592 (2014).

[6] B. Li, L. Wang, and G. Casati, Thermal Diode: Rectification of Heat Flux, Phys. Rev. Lett. 93, 184301 (2004).

[7] L. Wang and B. Li, Thermal Logic Gates: Computation with Phonons, Phys. Rev. Lett. 99, 177208 (2007).

[8] B. Hu, L. Yang, and Y. Zhang, Asymmetric Heat Conduction in Nonlinear Lattices, Phys. Rev. Lett. 97, 124302 (2006).

[9] J. Ordonez-Miranda, Radiative Thermostat Driven by the Combined Dynamics of Electrons, Phonons, and Photons, Phys. Rev. Appl. 14, 064043 (2020).

[10] M. Gandolfi, C. Giannetti, and F. Banfi, Temperonic Crystal: A Superlattice for Temperature Waves in Graphene, Phys. Rev. Lett. 125, 265901 (2020).

[11] J. Ordonez-Miranda, J. M. Hill, K. Joulain, Y. Ezzahri, and J. Drevillon, Conductive thermal diode based on the thermal hysteresis of $\mathrm{VO}_{2}$ and nitinol, J. Appl. Phys. 123, 085102 (2018).

[12] P. Ben-Abdallah and S.-A. Biehs, Phase-change radiative thermal diode, Appl. Phys. Lett. 103, 191907 (2013). 
[13] W. Kobayashi, Y. Teraoka, and I. Terasaki, An oxide thermal rectifier, Appl. Phys. Lett. 95, 171905 (2009).

[14] M. Terraneo, M. Peyrard, and G. Casati, Controlling the Energy Flow in Nonlinear Lattices: A Model for a Thermal Rectifier, Phys. Rev. Lett. 88, 094302 (2002).

[15] D. Segal, Single Mode Heat Rectifier: Controlling Energy Flow between Electronic Conductors, Phys. Rev. Lett. 100, 105901 (2008).

[16] A. Fiorino, D. Thompson, L. Zhu, R. Mittapally, S.-A. Biehs, O. Bezencenet, N. El-Bondry, S. Bansropun, P. BenAbdallah, E. Meyhofer, and P. Reddy, A thermal diode based on nanoscale thermal radiation, ACS Nano 12, 5774 (2018).

[17] I. Forero-Sandoval, J. Chan-Espinoza, J. Ordonez-Miranda, J. Alvarado-Gil, F. Dumas-Bouchiat, C. Champeaux, K. Joulain, Y. Ezzahri, J. Drevillon, C. Gomez-Heredia, and J. Ramirez-Rincon, $\mathrm{VO}_{2}$ Substrate Effect on the Thermal Rectification of a Far-Field Radiative Diode, Phys. Rev. Appl. 14, 034023 (2020).

[18] P. Ben-Abdallah and S.-A. Biehs, Near-Field Thermal Transistor, Phys. Rev. Lett. 112, 044301 (2014).

[19] J. Ordonez-Miranda, Y. Ezzahri, J. Drevillon, and K. Joulain, Transistorlike Device for Heating and Cooling Based on the Thermal Hysteresis of $\mathrm{VO}_{2}$, Phys. Rev. Appl. 6, 054003 (2016).

[20] H. Prod'homme, J. Ordonez-Miranda, Y. Ezzahri, J. Drevillon, and K. Joulain, Optimized thermal amplification in a radiative transistor, J. Appl. Phys. 119, 194502 (2016).

[21] I. Latella, O. Marconot, J. Sylvestre, L. G. Fréchette, and P. Ben-Abdallah, Dynamical Response of a Radiative Thermal Transistor Based on Suspended InsulatorMetal-Transition Membranes, Phys. Rev. Appl. 11, 024004 (2019).

[22] V. Kubytskyi, S.-A. Biehs, and P. Ben-Abdallah, Radiative Bistability and Thermal Memory, Phys. Rev. Lett. 113, 074301 (2014).

[23] K. Ito, K. Nishikawa, and H. Iizuka, Multilevel radiative thermal memory realized by the hysteretic metal-insulator transition of vanadium dioxide, Appl. Phys. Lett. 108, 053507 (2016).

[24] J. Ordonez-Miranda, Y. Ezzahri, J. A. Tiburcio-Moreno, K. Joulain, and J. Drevillon, Radiative Thermal Memristor, Phys. Rev. Lett. 123, 025901 (2019).

[25] F. Yang, M. P. Gordon, and J. J. Urban, Theoretical framework of the thermal memristor via a solid-state phase change material, J. Appl. Phys. 125, 025109 (2019).

[26] Z. Yu and S. Fan, Complete optical isolation created by indirect interband photonic transitions, Nat. Photon 3, 91 (2009).

[27] M. Maldovan, Sound and heat revolutions in phononics, Nature 503, 209 (2013).

[28] Y. Wang, B. Yousefzadeh, H. Chen, H. Nassar, G. Huang, and C. Daraio, Observation of Nonreciprocal Wave Propagation in a Dynamic Phononic Lattice, Phys. Rev. Lett. 121, 194301 (2018).

[29] G. Trainiti and M. Ruzzene, Non-reciprocal elastic wave propagation in spatiotemporal periodic structures, New J. Phys. 18, 083047 (2016).
[30] H. Nassar, X. Xu, A. Norris, and G. Huang, Modulated phononic crystals: Non-reciprocal wave propagation and willis materials, J. Mech. Phys. Solids 101, 10 (2017).

[31] R. Fleury, D. L. Sounas, and A. Alu, Subwavelength ultrasonic circulator based on spatiotemporal modulation, Phys. Rev. B 91, 174306 (2015).

[32] C. Shen, J. Li, Z. Jia, Y. Xie, and S. A. Cummer, Nonreciprocal acoustic transmission in cascaded resonators via spatiotemporal modulation, Phys. Rev. B 99, 134306 (2019).

[33] X. Zhu, J. Li, C. Shen, X. Peng, A. Song, L. Li, and S. A. Cummer, Non-reciprocal acoustic transmission via spacetime modulated membranes, Appl. Phys. Lett. 116, 034101 (2020).

[34] D. L. Sounas and A. Alu, Non-reciprocal photonics based on time modulation, Nat. Photon 11, 774 (2017).

[35] A. Mock, D. Sounas, and A. Alu, Magnet-free circulator based on spatiotemporal modulation of photonic crystal defect cavities, ACS Photonics 6, 2056 (2019).

[36] M. Camacho, B. Edwards, and N. Engheta, Achieving asymmetry and trapping in diffusion with spatiotemporal metamaterials, Nat Commun. 11, 3733 (2020).

[37] L. Xu and J. Huang, Thermal convection-diffusion crystal for prohibition and modulation of wave-like temperature profiles, Appl. Phys. Lett. 117, 011905 (2020).

[38] Y. Li, J. Li, M. Qi, C.-W. Qiu, and H. Chen, Diffusive nonreciprocity and thermal diode, Phys. Rev. B 103, 014307 (2021).

[39] D. Torrent, O. Poncelet, and J.-C. Batsale, Nonreciprocal Thermal Material by Spatiotemporal Modulation, Phys. Rev. Lett. 120, 125501 (2018).

[40] D. D. Joseph and L. Preziosi, Heat waves, Rev. Mod. Phys. 61, 41 (1989).

[41] R. Allan, Thermal-wave imaging, Science 218, 223 (1982).

[42] C. Vales-Pinzon, J. Ordonez-Miranda, and J. J. AlvaradoGil, Photothermal characterization of the thermal properties of materials using four characteristic modulation frequencies in two-layer systems, J. Appl. Phys. 112, 064909 (2012).

[43] M. A. Biot, Thermoelasticity and irreversible thermodynamics, J. Appl. Phys. 27, 240 (1956).

[44] S. Sun, S. Liang, W. C. Xu, G. Xu, and S. Wu, Photoresponsive polymers with multi-azobenzene groups, Polym. Chem. 10, 4389 (2019).

[45] J. Shin, J. Sung, M. Kang, X. Xie, B. Lee, K. M. Lee, T. J. White, C. Leal, N. R. Sottos, P. V. Braun, and D. G. Cahill, Light-triggered thermal conductivity switching in azobenzene polymers, PNAS 116, 5973 (2019).

[46] See Supplemental Material at http://link.aps.org/sup plemental/10.1103/PhysRevApplied.16.L041002 for the detailed homogenization of the temperature and heat flux profiles as well as the numerical solution of Eq. (1).

[47] J. Willis, Variational and related methods for the overall properties of composites (1981), p. 1.

[48] J. Willis, Variational principles for dynamic problems for inhomogeneous elastic media, Wave Motion 3, 1 (1981).

[49] F. Cervantes-Alvarez, J. D. Macias, and J. J. Alvarado-Gil, Heat transport in electrically aligned multiwalled carbon nanotubes dispersed in water, J. Phys. D: Appl. Phys. 51, 065302 (2018). 